\title{
Deviation of Pedestrian Path due to the Presence of Building Entrances
}

\author{
Shi Sun $\mathbb{D}^{1,2}$ Cheng Sun, ${ }^{1,2}$ Dorine C. Duives $\mathbb{D}^{3},{ }^{3}$ and Serge P. Hoogendoorn $\mathbb{D}^{3}$ \\ ${ }^{1}$ School of Architecture, Harbin Institute of Technology, Harbin 150006, China \\ ${ }^{2}$ Key Laboratory of Cold Region Urban and Rural Human Settlement Environment Science and Technology, \\ Ministry of Industry and Information Technology, Harbin 150006, China \\ ${ }^{3}$ Department of Transport \& Planning, Faculty of Civil Engineering and Geosciences, Delft University of Technology, \\ CN Delft 2628, Netherlands
}

Correspondence should be addressed to Shi Sun; sunshi_1989@163.com

Received 10 February 2021; Revised 6 May 2021; Accepted 15 June 2021; Published 30 June 2021

Academic Editor: Ren-Yong Guo

Copyright (C) 2021 Shi Sun et al. This is an open access article distributed under the Creative Commons Attribution License, which permits unrestricted use, distribution, and reproduction in any medium, provided the original work is properly cited.

Commercial areas, especially urban ones with numerous buildings, are becoming increasingly prone to congestion because of their popularity. Visual inspections show that interactions between pedestrians and building entrances affect the distribution of pedestrian trajectories, which influences the utility of pedestrian spaces and the design of urban shopping areas. Herein, we analyse the dynamics of pedestrian deviations around building entrances. We used a video recorded using an unmanned aerial vehicle to determine pedestrian trajectories in a Chinese commercial walking space. First, the candidate variables affecting deviation behaviours were determined via correlation testing. Second, two regression models were developed by considering the deviation behaviours of pedestrians walking past a building entrance. The models suggest that the starting position of a pedestrian's deviation, the total pedestrian flow at the building entrance, the density in an area in the vicinity of the entrance, and the number of interacting pedestrians impact the total distance traversed during path deviation.

\section{Introduction}

Business districts have become spaces where people enjoy leisure time, pedestrians gather, and commercial activities are carried out. To improve the shopping experience, many central urban spaces have been converted for pedestrian-only use. In these spaces, commercial activities compete to attract consumers, which causes increasing numbers of pedestrians to gather in commercial areas, particularly during weekends and holidays $[1,2]$. However, these crowded walking environments diminish the shopping experience and can potentially trigger hazardous crowd incidents $[3,4]$.

Pedestrian flow models are often used to analyse and predict the movement dynamics in pedestrian spaces. These models are generally categorised as macroscopic models-such as the continuum models and network models-or microscopic models-such as the social force, velocity obstacle, and discrete choice models. In principle, it is expected that microscopic models exhibit better performance in terms of the accuracy of an agent's movement dynamics, whereas macroscopic models generally require less computational effort $[5,6]$.

The social force model is well-studied and a recent microscopic extension that considers both physical factors and motivational factors is often used to analyse crowd behaviour [7]. According to the social force model principles, pedestrian movement is determined by the internal force required to walk at a desired speed, the movements of other pedestrians, and the boundaries of objects [8]. In general, the shortest path to an agent's destination, an agent's preferred walking speed, the movement behaviours of the surrounding pedestrians, and the presence of obstacles (e.g., dustbins, benches, and building facades) are considered when modelling commercial pedestrian areas.

In building areas, pedestrians interact with other pedestrians in open space and with pedestrians entering and 
exiting building entrances, where orthogonally intersecting flows are encountered. At such locations, passing pedestrian flows are potentially affected by the flow of pedestrians entering and exiting the building entrances and the building facades [9]. In most contemporary pedestrian models featuring commercial areas, the effect of (entrances in) building facades on movement behaviour is not considered. Because building entrances are important features of commercial districts and the entrances potentially influence expectations regarding capacity and the levels-of-service available in commercial pedestrian spaces, it is imperative to understand the impact of the dynamic connection between building entrances and pedestrian movement more clearly.

This study aims to determine the extent to which the trajectories of passing pedestrians are affected by the presence of an entrance in a building facade. The most important factors affecting the lateral and longitudinal deviation distance of pedestrians passing in front of building entrances are to be determined. The movement behaviours of pedestrians featuring in unmanned aerial vehicle (UAV) video recordings of a Chinese commercial area were analysed to determine the trajectories of pedestrians near entrances. Next, using a set of one-to-one correlation tests and a linear regression model, the most significant factors affecting the lateral and longitudinal deviations were determined.

This paper first provides a brief overview of the previous research focusing on pedestrian movement dynamics. Next, the research methodology is elaborated upon. Accordingly, a case study area is presented. The results of the one-to-one correlation tests and the linear regression modelling are subsequently described. Finally, several conclusions and a discussion of the implications of the result considering the design of commercial pedestrian areas are presented.

\section{Background}

This section briefly reviews factors in the pedestrian or walking environment that can affect pedestrian walking behaviour. The literature can be divided into works that feature the impact of personal characteristics, the interaction with other pedestrians, and the interaction with the built environment, which are factors that influence pedestrian walking dynamics.

2.1. Impact of Personal Characteristics. Individual factors of pedestrians can impact the walking behaviour of pedestrians. Finnis and Walton [10] and Duives, Daamen, and Hoogendoorn [11] studied the connection between pedestrians' walking speed and several individual characteristics. They found that gender, age, physique, goal orientation, presence of children, baggage, shoe-type, and looking around behaviour significantly correlated with walking speed. Other works established the impact of culture [12-14] and group composition [15-18].

2.2. Interactions with Other Pedestrians. Various research groups have studied the interaction between pedestrians, especially in unidirectional single file, unidirectional bottleneck, and bidirectional movement scenarios. Seminal works by Hankin and Wright [19], Weidmann [20], Daamen and Hoogendoorn [21], and Seyfried et al. [22] provided first insights into the impact of pedestrian movement dynamics on the capacity of a space. In general, these studies illustrate that crowd density negatively impacts the walking speed of pedestrians. Zacharias [23] found that the presence of pedestrians can improve the appeal of a location, and $\mathrm{Qu}$ et al. [24] found that pedestrians will avoid overcrowded areas. More recently, Kretz et al. [25]; Wong et al. [26]; Duives, Daamen, and Hoogendoorn [6]; and Cao et al. [27] studied the pedestrian flow under more diverse conditions, such as bidirectional straight flows and those that intersect. It was found that more complex flow situations and the resultant collision avoidance behaviours reduce the maximum sustainable flow rate in the pedestrian infrastructure.

Thus, when modelling pedestrian movement dynamics, the crowd's density and the interactions between pedestrians should be included. In cluttered walking environments, pedestrians veer to avoid potential collisions [28], but the extent to which such deviations are affected by other pedestrians remains unclear.

2.3. Interaction with the Built Environment. Pedestrians also react to the physical layout of a space. In commercial pedestrian areas, buildings and sidewalks are two of the dominant physical features that pedestrians interact with. These interactions can be classified as accessibility and visibility [29].

2.3.1. Buildings. Pedestrians' operational walking dynamics might alter near "obstacles." Walls, which pedestrians avoid to mitigate the risk of getting hurt, function as the physical boundaries of the walking environment [30]. Moussaid et al. [31] experimentally studied the walking trajectories when passing a corridor and established that pedestrians prefer to maintain a distance of at least $30 \mathrm{~cm}$ from a wall. Furthermore, Ma et al. [32] showed that for Asian people, the shy-away distance is slightly larger and the repulsion force is less.

Buildings affect pedestrian movement dynamics in a variety of ways. First, pedestrians decide to move towards a certain destination. Oppewal and Timmermans [33] indicate that the appeal of the area, and thus, the choice to visit it, depends strongly on the maintenance of the whole and the appearance of shop fronts, as well as street activities, greenery, coffee shops, and the presence of crowds. Meziani and Hussien [34] illustrated that 12 factors increase the appeal of commercial activities, among these factors, toilets and prayer rooms, good ambience, parking, mall location, variety of brands, and family activities were considered to be the top indicators. Moreover, building facades block the sight of pedestrians, limiting their visual scope. Jansen, Toet, and Werkhoven [35] found that when the visual scope of pedestrians is limited, they deviate to avoid potential danger. 
2.3.2. Sidewalk. Distinct from buildings, sidewalks are exterior urban walking spaces that pedestrians directly interact with. Normally, a sidewalk in the built environment consists of several elements, which contribute to the area's complexity. Fajen and Warren [36] studied the intensities of attraction and repulsion in complex environments and established that the strength of the attraction/repulsion response to objects depends on the distance separating them from the pedestrian.

Zacharias [23] studied how pedestrians respond to the public walking environment and decide their walking routes. It was found that the visual impression of infrastructures is important for attracting pedestrians to visit. Chen [37] classified the environment of the pedestrian street into three categories-the fixed-feature elements (facades, furniture on the street, street lamps, etc.), the semifixed-feature elements (shop signs, plants, displays, outdoor cafes, food stands, etc.), and nonfixed-feature elements (pedestrians) - and found that street furniture, street performance, and shop signage can attract pedestrians and divert crowds. Simultaneously, however, these elements can also act as obstacles and repel pedestrians. Matthis and Fajen [38] and Severiukhina et al. [39] studied pedestrian behaviour that is influenced by obstacles in a complex walking environment and found that influenced by various obstacles, pedestrian walking speed will decrease when there are more potential collisions.

2.4. Pedestrian Behaviour around Building Entrances. As the connections between buildings and sidewalks, spaces near entrances are more complex and variously affect pedestrians' behaviour. Previous studies attended mainly to the inflow and outflow to and from entrances and particularly studied the flow dynamics through the entrance [40]. The building entrance influences the entering and exiting of pedestrians but the shape of the connections between the exterior and interior spaces could also influence the movement dynamics of those that walk parallel to the building facade. It was found that factors such as aperture width, corridor length, and transparency of the doors could affect the behaviour of passing pedestrians [41]. Influenced by the attraction of commercial activities, pedestrians show waiting [42], wandering [37], entering, and exiting behaviour [43], which contributes to a chaotic walking environment and diverts the passage of pedestrians. Moreover, because building facades will limit the visual scope of passing pedestrians, the walking dynamics will be affected because visual information is lacking. Consequently, the presence of entrances should be acknowledged when modelling pedestrian walking dynamics near building facades. However, to the authors' knowledge, the impact of entrances in the immediate vicinity on pedestrian walking dynamics is undetermined.

\section{Research Methodology}

We modelled the impact of an entrance on pedestrian walking dynamics. First, the deviating movements of pedestrians were parametrised. Subsequently, the factors that potentially affect the deviations were determined. Finally, a procedure was established to identify the most significant factors that affected pedestrian deviation behaviours near entrances.

\subsection{Parameterising Deviation Movement of Pedestrians.} This section describes a methodology to quantify the deviation behaviours of pedestrians. Between entering and leaving a region, a pedestrian's path includes five key points at which direction may be changed (Figure 1). The first point, where a pedestrian enters the area is $P_{o} ; P_{s}$ is the point where the deviation starts; $P_{m}$ is the point of maximum deviation from the original trajectory; $P_{f}$ is the point where the path is resumed; $P_{e}$ is the pedestrian's point of exiting the area (Figure 1). This study focuses on the section between $P_{s}$ and $P_{m}$, which determines the lateral $(\Delta y)$ and longitudinal $(\Delta x)$ distances traversed after deviating from the original trajectory near an entrance. The criteria for identifying $P_{s}$ and $P_{m}$ are introduced below.

3.1.1. Starting Point of Deviation $\left(P_{s}\right)$. Four criteria are considered for identifying the position $\left(x_{o}, y_{o}\right)$ of $P_{s}$. The first criterion is the point's location, that is, the point $P_{s}$ should be located before the entrance and within $6-24 \mathrm{~m}$ before the maximum-deviation point on the $x$-axis to confirm that the entrance caused the deviation and that the correlation between these two points is within a reasonable range. The following two criteria filter out the adjustment of path and speed caused by collision avoidance behaviour [44]. The second criterion is the walking direction. To guarantee a stable deviation trend, the pedestrian should continue away from the entrance from the point $P_{s}$ for at least the next $5 \mathrm{~s}$. The third criterion is angular speed, which should be $>0$ for the next $1.5 \mathrm{~s}$ after the point $P_{s}$. Moreover, during this period, the angular speed must be $>0.02 \mathrm{~m} / \mathrm{s}^{2}$ for at least $1 \mathrm{~s}$ to verify that the turning is sufficiently noticeable. Finally, there should be no other viable starting points farther away from the wall than the maximumdeviation point between $P_{s}$ and $P_{m}$; if such a starting point exists, the point farther away will become the maximumdeviation point.

3.1.2. Maximum Deviation Point $\left(P_{m}\right)$. Four criteria were adopted to identify $P_{m}$. The first criterion was its position, which should be within $15 \mathrm{~m}$ from the entrance because it is difficult to confirm the reason for deviations that begin distantly from the entrance. Second, pedestrians should continue moving away from the wall in the $4 \mathrm{~s}$ before reaching point $P_{m}$ and should move closer to the wall in the $3 \mathrm{~s}$ after the crossing point $P_{m}$. This criterion ensures that minor fluctuations are eliminated. The third criterion is the angular speed, which should be $<0$ for at least $1 \mathrm{~s}$ before and after point $P_{m}$ to ensure that the critical point is sufficiently important in the process. Similar to the case of point $P_{s}$, no other viable maximum-deviation points should be located further away from the wall than the current $P_{m}$. 


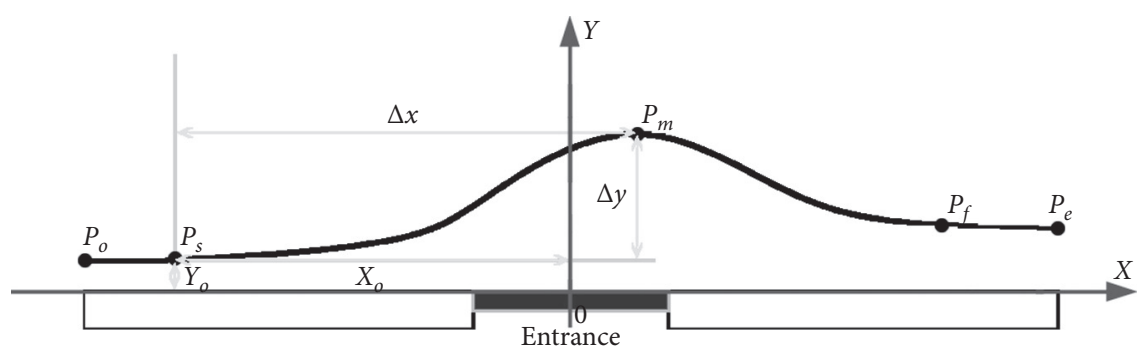

Figure 1: Key points of passing trajectories.

3.2. Objectifying Potential Impact Factors. Based on the literature review presented above, four types of variables are identified and used to predict the subject's lateral and longitudinal deviation distance: the deviation's starting position, the flow through the entrance, the pedestrian density in the immediate vicinity of the entrance, and type of pedestrian flow within a certain range from the subject.

To quantify the interaction with the built environment, the starting position of the deviation is defined to describe the position relative to the building facade and entrance when the deviation starts. The interactions with other pedestrians are classified as either influenced by the entrance or influenced by self-organisation behaviour. The cluttered environment caused by the appeal of commercial activity is quantified by flow through the entrance and pedestrian density in the direct vicinity of the entrance. The influence from self-organisation behaviour is quantified by pedestrians walking in different directions within the interaction range and in front of the specific pedestrian. The first type of variable has previously been defined above. The second to fourth variables are described in more detail in the following subsections.

3.2.1. Entrance Flow. The pedestrian flow at the entrance is quantified as the average number of pedestrians who walk in or out of a shopping entrance during the period in which a pedestrian of focus (subject) approaches the entrance. In this study, several entrance flow variables were determined, each within a slightly different range of the entrance (Figure 2(a)). The starting point of this range is between $-20 \mathrm{~m}$ and $-10 \mathrm{~m}$, and the interval for choosing variables is $2 \mathrm{~m}$, meaning that the starting and finishing points are even-numbered. The total entrance flow $(F)$ consists of two categories: pedestrians who pass through the entrance while maintaining their current direction are the inflow, and those continuously moving away in the opposite direction are the outflow. For a pedestrian to be considered in the inflow or outflow, the walking speed must be greater than $0.68 \mathrm{~m} / \mathrm{s}$ [45]. That is, pedestrians who stop in front of the entrance are not considered. Based on the starting position $(a)$ and the finishing position $(b)$ of the range, the variables of the entrance pedestrian flow are the sum of the inflow and outflow $\left(F_{a}^{b}\right)$.

3.2.2. Entrance Density. The entrance density is the average value of the density in a certain area in the vicinity of the entrance when the subject is between the starting $\left(P_{s}\right)$ and endpoints $\left(P_{m}\right)$ of the deviation (Figure 2(b)). A rectangular area with a lateral range of $9 \mathrm{~m}$ is adopted. The lateral range varies between $-10 \mathrm{~m}$ and $10 \mathrm{~m}$ along the $x$-axis, and the step size between the entrance-density variables is $2 \mathrm{~m}$. The variable of the entrance density is $E_{a}^{b}$, where $a$ indicates the $x$ coordinate at the start of the longitudinal range and $b$ the $x$ coordinate at the end of the longitudinal range.

3.2.3. Number of Interacting Pedestrians. The number of interacting pedestrians is defined as the average value of the number of pedestrians that fulfill three criteria (Figure 2(c)). First, the position of the interacting pedestrians should be within the interaction range $(4.5 \mathrm{~m})$ from the subject [46]. In the following two criteria, if the angle points in an anticlockwise direction relative to the positive direction of the $x$ axis, the numerical value is positive. Otherwise, the angle is negative. Second, the angle between the positive direction of the $x$-axis and the relative location of the pedestrian interacting with the subject should be within a certain threshold. The region from $-90^{\circ}$ to $90^{\circ}$ in the positive direction of the $x$-axis from the subject is divided into six parts $\left(-90^{\circ}\right.$ to $-60^{\circ},-60^{\circ}$ to $-30^{\circ},-30^{\circ}$ to $0^{\circ}, 0^{\circ}$ to $30^{\circ}, 30^{\circ}$ to $60^{\circ}$, and $60^{\circ}$ to $90^{\circ}$ ), and pedestrians within the specific region are selected. The lower and upper boundaries of the region are recorded as $\alpha$ and $\beta$, respectively $(\alpha<\beta)$. Third, the angle between the positive direction of the $x$-axis and the interacting pedestrian's direction of movement should be within a certain range. The $-180^{\circ}$ to $180^{\circ}$ range of the interacting pedestrian's movement direction with the positive direction of the $x$-axis is divided into six sectors $\left(-180^{\circ}\right.$ to $-120^{\circ},-120^{\circ}$ to $-60^{\circ},-60^{\circ}$ to $0^{\circ}, 0^{\circ}$ to $60^{\circ}, 60^{\circ}$ to $120^{\circ}$, and $120^{\circ}$ to $180^{\circ}$ ). Then, pedestrians with the direction of movement within the specific sector are selected. The lower and upper limits of the sector are recorded as $\gamma$ and $\theta$, respectively $(\gamma<\theta)$. Using $\alpha$, $\beta, \gamma$, and $\theta$, the numbers of interacting pedestrians can be designated using the following naming convention $P_{\alpha, \beta}^{\gamma, \theta}$.

3.3. Modelling Pedestrian Deviation Behaviour. A two-step process is adopted to determine the factors that affect the deviation behaviour. This process features a set of one-toone correlation tests and stepwise linear regression modelling. Both these steps are briefly elaborated upon in the following subsections.

3.3.1. One-to-One Correlation Test. Numerous potential variables have been defined. To preselect the variables that potentially have a strong correlation with the lateral or 


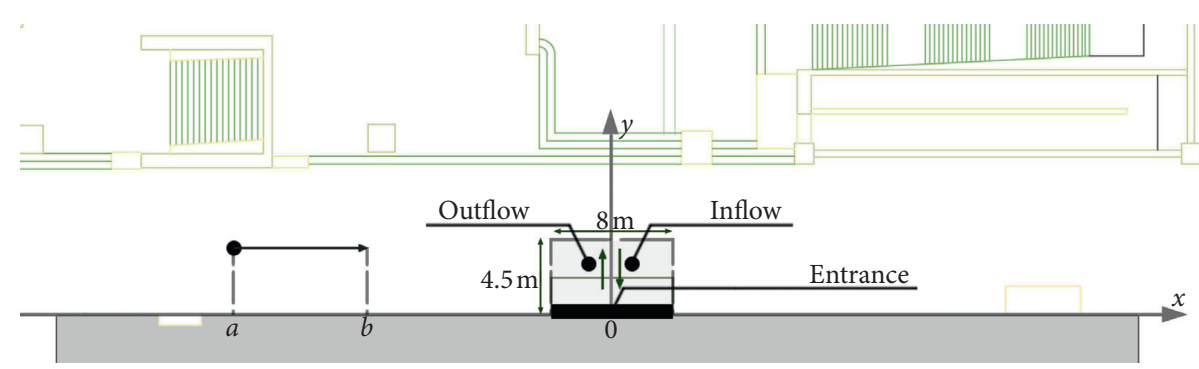

(a)

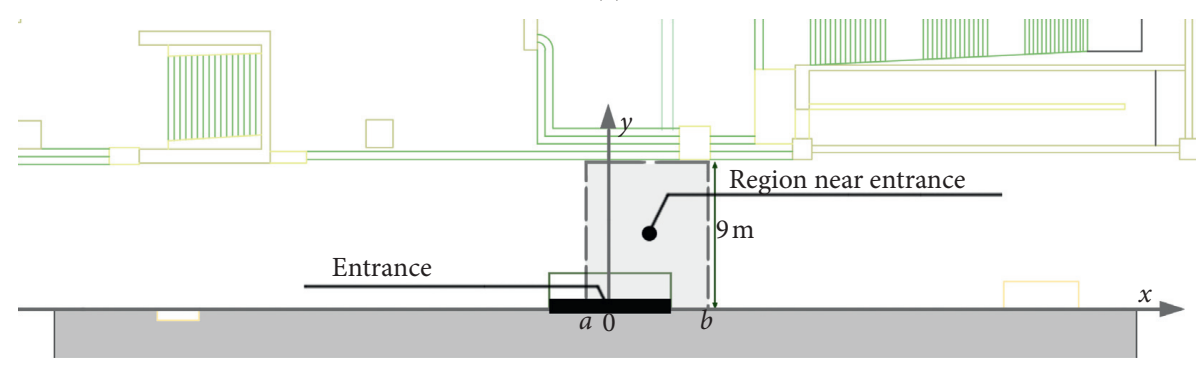

(b)

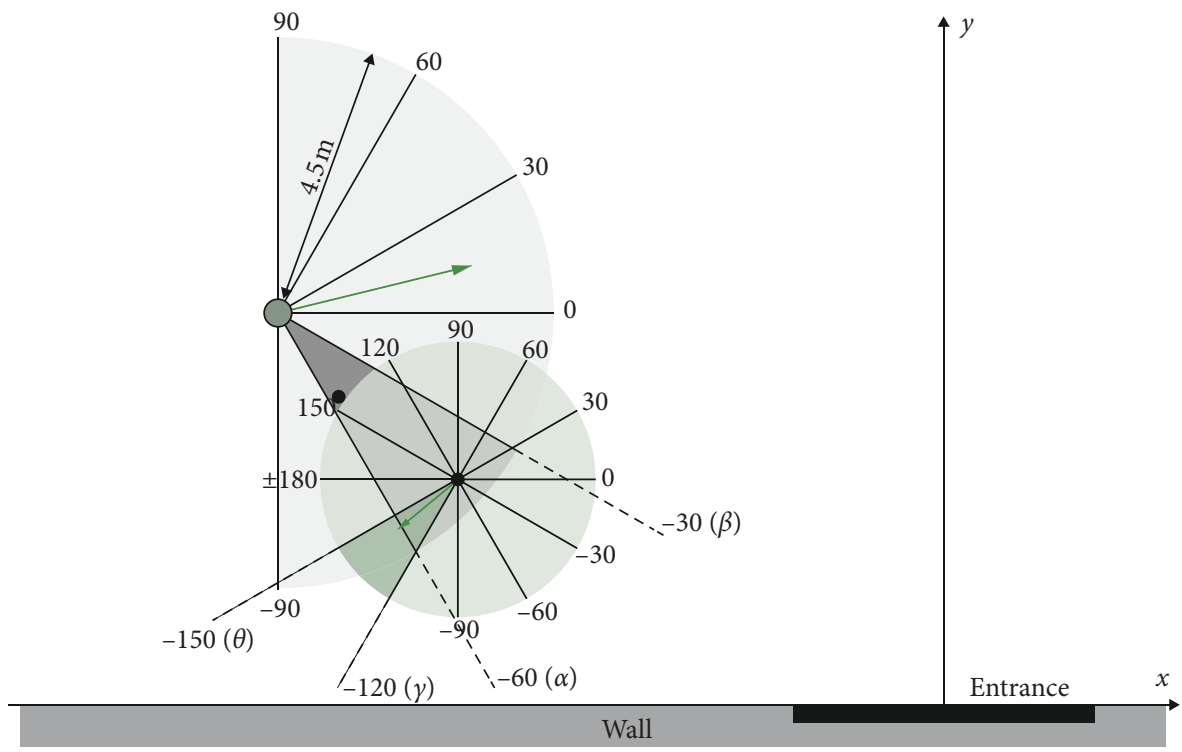

(c)

Figure 2: The variables used to quantify the movement dynamics in front of the entrance of a building and near a subject; the entrance is indicated by a black bar and the subject is indicated by a green circle. (a) Entrance flow. (b) Entrance density. (c) Interacting pedestrians.

longitudinal deviation, the correlation between every variable and each of the two dependent variables is established using a paired $t$-test from the Statistical Package for the Social Sciences (SPSS). Here, the $p$ value is the selection criterion. All variables that have a strong correlation with the dependent variable $(p<0.20)$ are selected for the second step of the modelling process.

3.3.2. Stepwise Regression. In the second step, stepwise linear regression modelling is used. This procedure in SPSS automatically selects the best variables for the linear regression model. First, the most significant variable is entered. Then, in every step, all new combinations of $n+1$ variables are attempted, and the combination with the highest increase in explanatory power is retained. Second, the added explanatory power of all the current variables in the model is checked. A variable is erased when its added explanatory power is excessively low. This process is repeated until no more variables can be entered or erased without improving or compromising the explanatory power of the model. In this case, the explanatory power of a variable to be added is 0.009 , and that of a variable not to be erased is 0.010 .

\section{Case Study: Chinese Commercial Area}

As pedestrians in the target area of study exhibit a variety of behaviours, which creates a complex walking environment. Pedestrian behaviour is difficult to control in the complex 
scenarios used for experimentation [47], so the field observation data-collection method is used in this study. A case study was conducted in a Chinese commercial pedestrian area. The case study area is first presented then the dataprocessing and filtering procedures are discussed.

4.1. Description of the Scene. A video was recorded on Saturday, September 1, 2018, a sunny day, which made shopping and visiting the square a pleasant experience. The video was recorded using a video camera attached to a DJI Mavic Pro (i.e., a light UAV weighing 743 g). The camera has a resolution of $2720 \times 1536$ pixels and a frame rate of $24 \mathrm{fps}$. During recording, the flying height was $90 \mathrm{~m}$, which is sufficient to observe both ends of the passage and to observe the movement of pedestrians clearly. Because of the camera pan, the averaged measurement error in every $0.5 \mathrm{~s}$ diminishes to 0.4641 pixels $(0.0182 \mathrm{~m})$. The recording lasted for $10 \mathrm{~min}$, from $1: 45$ p.m. to $1: 55$ p.m.

The case study area was a busy commercial district near the flood-control victory monument on Harbin Central Street, China. The upper part of the area is a shopping mall with an entrance in the middle of the building facade and a restaurant entrance at either end of the facade. The lower part of the area consists of steps leading to underground passages (Figure 3). The area between the building and the underground passages is $60 \mathrm{~m}$ long and $9 \mathrm{~m}$ wide. Within this space, there are six dominant starting points and destinations, which are at the left side, bottom, right side, and entrance of the shopping mall, and the two restaurant entrances. In this study, the movement behaviour in front of the shopping mall entrance was mainly investigated because very few pedestrians enter and exit the restaurants, and the location of two restaurant entrances $(24.5 \mathrm{~m}$ from the centre of the commercial entrance) is beyond the region that deviation behaviour appears. The height of handrails is not enough to block the pedestrians' line of sight. Thus, the commercial entrance of the building is the only environmental factor that attracts pedestrians and blocks their line of sight.

Most people in the case study area were young or middle-aged and walked normally towards their destination. Most tended to alter direction near the entrance and move away from the wall. After passing the entrance region, they tended to change direction again and move back to their original trajectory.

The video analysis software "Tracker," which is a program built on an open-source physics Java framework, was used to record the trajectories of all pedestrians within the case study area. The origin of the coordinate system, the positive direction of the $x$-axis, and the unit length can be fixed against the unchanged background in the video (the building facade in this study), which can further reduce the averaged measurement error. The movements of the upper body were a part of the captured trajectories. After a semiautomatic tracking procedure, the remaining tracking errors produced by the software were corrected manually upon visual inspection.

4.2. Data Processing and Filtering. Every trajectory consists of a sequence of positions $(x, y, t)$, which were extracted every two frames (12 fps). The centre of the building was adopted to transform the trajectory coordinates from pixel coordinates to real-world coordinates. To ensure that all pedestrians were on the positive side of the $y$-axis, the scene was inverted vertically. Moreover, to ensure that all pedestrians traversed in the positive direction along the $x$-axis (Figure 4(a)), the $x$-axis was switched for trajectories approaching from the right (Figure 4(b)).

In this study, we primarily focused on the movement dynamics of pedestrians passing in front of the entrance but not entering or standing still in the vicinity of the entrance. Thus, from the total set of all trajectories (1565), a four-step filtration process was applied to select only pedestrians who passed the entrance (Figure 5) to form the subject set used in the modelling process. The four steps are described below:

(i) Pedestrians have to pass through the region in front of the entrance $(-20 \mathrm{~m}<x<20 \mathrm{~m})$

(ii) Pedestrians should always be inside the analysis region

(iii) Pedestrians should have a "normal" walking speed $(0.68 \mathrm{~m} / \mathrm{s}<x<1.92 \mathrm{~m} / \mathrm{s})$ [45]

(iv) Pedestrians should deviate from their path near the entrance

Figure 5 shows the trajectories that were discarded from this set in each step of the filtration process. Of the 1565 trajectories, two pedestrians traversed the research area from right to left and returned from left to right and their movements were recorded as two trajectories. Using these filtration steps, 91 trajectories were considered in the analysis.

4.3. Trends in the Data. The mean $y$-coordinate value for every $0.5 \mathrm{~m}$ along the $x$-axis of all the deviating pedestrians was calculated, and the trend of deviation can be visualised as a plot (Figure 6). Pedestrians passing the entrance showed a lateral deviation of $2.148 \mathrm{~m}$ on average, with a standard deviation of $1.201 \mathrm{~m}$. The mean value of longitudinal deviation was $13.257 \mathrm{~m}$, with a standard deviation of $5.160 \mathrm{~m}$.

Note that pedestrians had passed the central position of the entrance when they reached the maximum-deviation point, which could be caused by two reasons. First, the deviation trend would not stop immediately because of the inertia of pedestrian dynamics [48]. Second, when passing pedestrians reached the central position of the entrance, pedestrians on the side near the building still exhibited a variety of behaviours, which continued creating a cluttered environment that diverted pedestrian movement. 


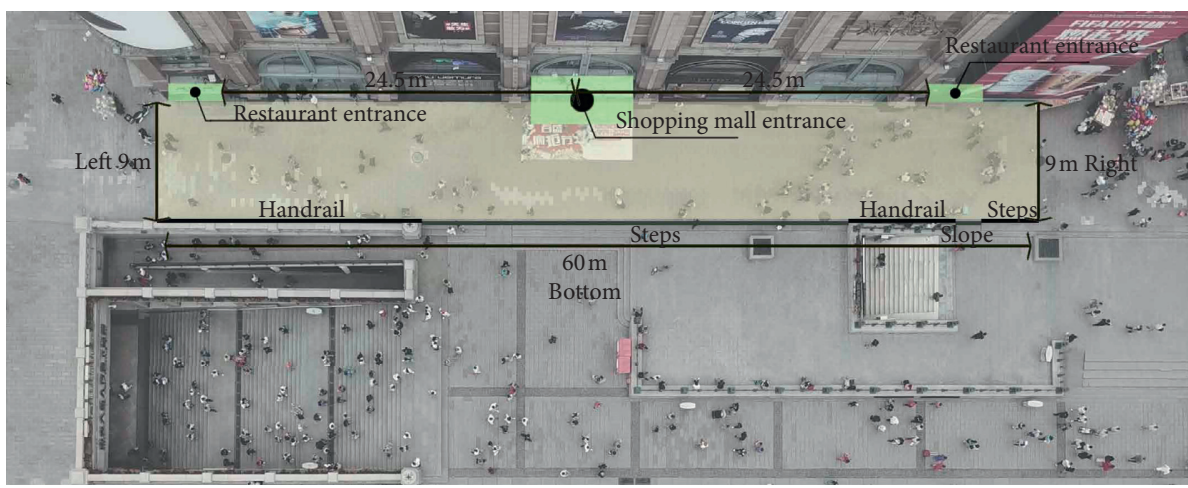

FIGURE 3: Snapshot of the UAV video footage, where the yellow area is the case study area.
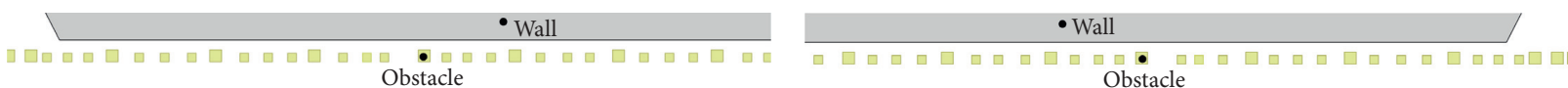

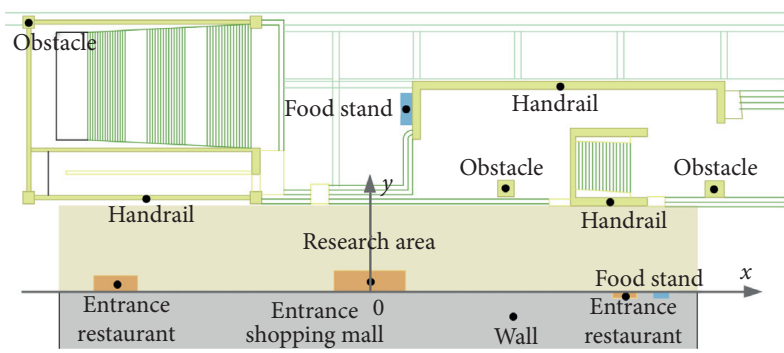

(a)

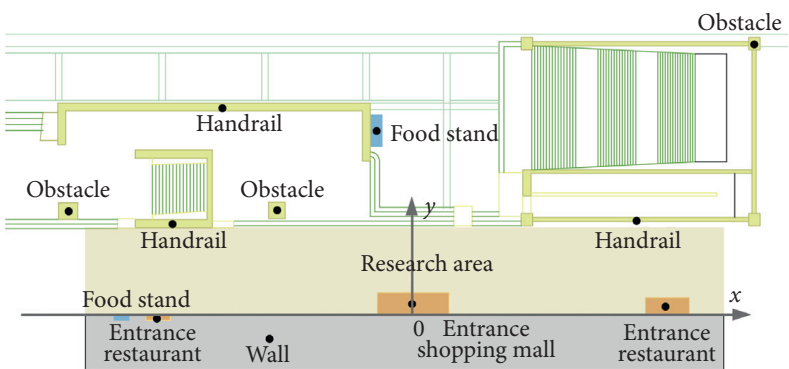

(b)

Figure 4: Schematic of the coordinate system. (a) Coordinate system for pedestrians coming from the left. (b) Coordinate system for pedestrians coming from the right.

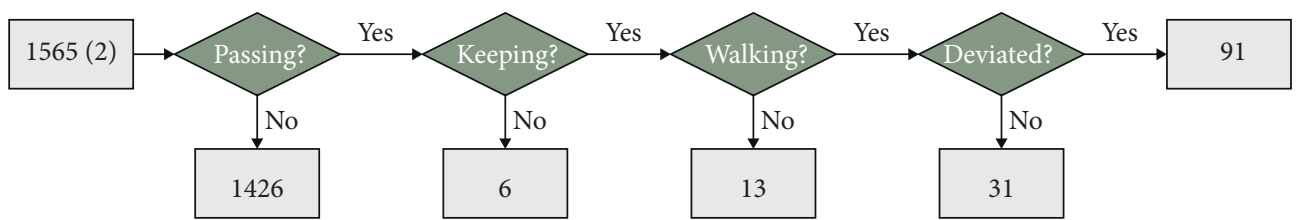

FIGURE 5: Trajectory filtering processing procedure.

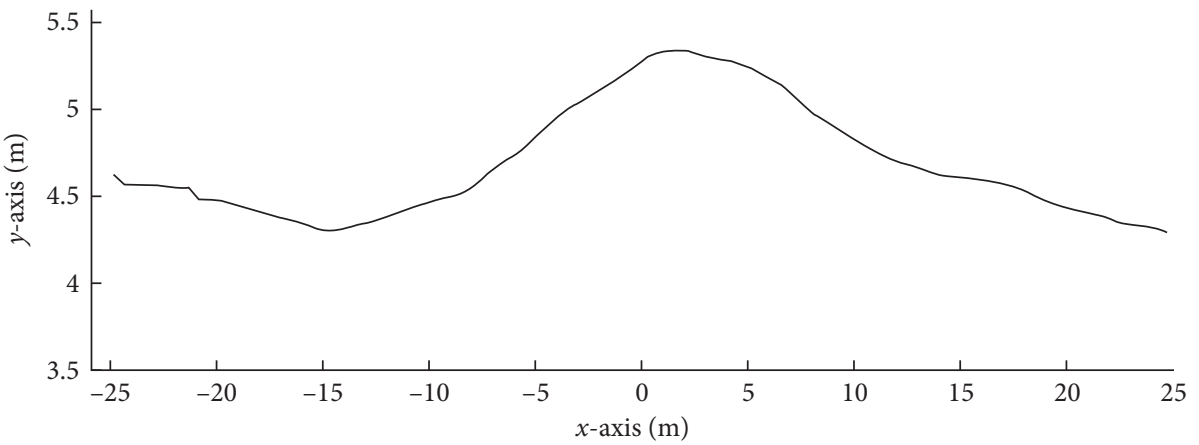

Figure 6: Plot of the mean value of all deviated trajectories. 


\section{Result}

\subsection{One-to-One Correlation}

5.1.1. The Correlation of the Deviation Process. We showed that $y_{o}$ (with a mean value of $3.813 \mathrm{~m}$ and a standard deviation of $1.492 \mathrm{~m}$ ) was correlated with $\Delta y$ but not with $\Delta x$; thus, the distance to the wall when the deviation starts is related to the lateral deviation, as summarized in Table 1. $x_{o}$ (with a mean value of $-11.550 \mathrm{~m}$ and a standard deviation of $5.562 \mathrm{~m}$ ) is related to $\Delta x$; however, its relationship with $\Delta y$ is less apparent. In this sense, the longitudinal deviation is correlated with the distance to the entrance on the $x$-axis when the deviation begins. The correlation of $\Delta y$ with $\Delta x$ is strong.

5.1.2. The Correlation of Entrance Flow. In this study, the mean value of pedestrian flow is 2.578 persons, with a standard deviation of 2.072 persons. Entrance flow is correlated with $\Delta y$ and $\Delta x$, as shown in Figure 7 . The pedestrian flow when the subject is closer to the entrance has a strong correlation with $\Delta y$ and $\Delta x$. The $p$ values of $F_{-16}^{-4}, F_{-14}^{-4}, F_{-12}^{-4}$, and $F_{-10}^{-4}$ with $\Delta y$ are $0.009,0.001,0.000$, and 0.000 , respectively, which indicates a strong correlation, showing that the lateral deviation of pedestrians at a distance of less than $16 \mathrm{~m}$ from the entrance will be influenced by the entrance flow. The $p$ value of $F_{-20}^{-6}, F_{-20}^{-4}, F_{-18}^{-6}, F_{-18}^{-4}, F_{-16}^{-6}, F_{-16}^{-4}, F_{-14}^{-8}$, $F_{-14}^{-6}, F_{-14}^{-4}, F_{-12}^{-6}, F_{-12}^{-4}$, and $F_{-10}^{-4}$ with $\Delta x$ is less than 0.020 . This shows that the entrance flow's scope of influence on longitudinal deviation can be greater than on lateral deviation. Then, the longitudinal deviation depends on the mean pedestrian flow of a large time interval, but the extent of deviation still depends on the degree of activity when the passing pedestrian approaches the entrance area.

5.1.3. The Correlation of Entrance Density. The entrance density is correlated with $\Delta y$ and $\Delta x$, as shown in Figure 8. $E_{-10}^{-4}$ (with a mean value of 0.219 persons $/ \mathrm{m}^{2}$ and a standard deviation of 0.073 persons $/ \mathrm{m}^{2}$ ) has a strong correlation with $\Delta y$ having the $p$ value of $0.010 ; E_{-4}^{6}$ (with a mean value of 0.275 persons $/ \mathrm{m}^{2}$ and a standard deviation of 0.053 persons/ $\mathrm{m}^{2}$ ); $E_{-4}^{8}$ (with a mean value of 0.264 persons $/ \mathrm{m}^{2}$ and a standard deviation of 0.050 persons $/ \mathrm{m}^{2}$ ); $E_{-2}^{8}$ (with a mean value of 0.253 persons $/ \mathrm{m}^{2}$ and a standard deviation of 0.051 persons $/ \mathrm{m}^{2}$ ); $E_{0}^{8}$ (with a mean value of 0.252 persons $/ \mathrm{m}^{2}$ and a standard deviation of 0.059 persons $/ \mathrm{m}^{2}$ ); and $E_{2}^{8}$ (with a mean value of 0.242 persons $/ \mathrm{m}^{2}$ and a standard deviation of 0.064 persons $/ \mathrm{m}^{2}$ ) have strong correlations with $\Delta y$ having the $p$ values of $0.017,0.011,0.016,0.016$, and 0.019 , respectively. The density in the area upstream of the entrance's central point shows a stronger correlation with $\Delta y$, and the density of the area a little after shows a stronger correlation with $\Delta x$. Passing pedestrians will be influenced more by the pedestrian density upstream of the entrance and decide the extent of lateral deviation to be away from the specific complex walking environment. The pedestrian density behind the central point of the entrance will influence when the passing pedestrians will return to the desired path.
5.1.4. Correlation of the Number of Interacting Pedestrians. For all the variables showing the number of interacting pedestrians, the mean value is 0.196 , with a standard deviation of 0.314 . It can be found that the number of interacting pedestrians is correlated with $\Delta y$ and $\Delta x$, as shown in Figure 9. $P_{-90,-60}^{-150,-90}, P_{-90,-60}^{90,150}, P_{-60,-30}^{-150,-90}, P_{-30,0}^{-150,-90}$, and $P_{0,30}^{-150,-90}$ are found to strongly correlate with $\Delta y$ with $p$ values of $0.005,0.018,0.018,0.010$, and 0.000 , respectively, while $P_{-90,-60}^{-30,30}$ is strongly correlated with $\Delta x$ with the $p$ value of 0.005 . The lateral deviation was found to be more sensitive to collisions with pedestrians walking towards the wall, and the longitudinal deviation varied with the number of pedestrians walking along the wall on the near side.

\subsection{Regression Model}

5.2.1. Lateral Deviation. The 11 variables found to be strongly correlated with $\Delta y$ are $y_{o}, F_{-16}^{-4}, F_{-14}^{-4}, F_{-14}^{-4}, F_{-10}^{-4}$, $E_{-10}^{-4}, P_{-90,-60}^{-150,-90}, P_{-90,-60}^{90,150}, P_{-60,-30}^{-150,-90}, P_{-30,0}^{-150,-90}$, and $P_{0,30}^{-150,-90}$. After stepwise regression, $y_{o}, F_{-10}^{-4}$, and $P_{0,30}^{-150,-90}$ were incorporated into the regression model for predicting lateral deviation, as shown in (1).

The regression model shows that $\Delta y$ is negatively correlated with $y_{o}$ (with a $t$ value of -1.791 and a $p$ value of 0.001), which indicates that if the starting position of the subject is closer to the wall, the lateral deviation distance is larger. This implies that the visual scope of pedestrians walking nearer to the wall is blocked to a greater degree and a further lateral deviation is needed to avoid potential hazards. $\Delta y$ has a positive correlation with $F_{-10}^{-4}$ (with a $t$ value of 4.500 and a $p$ value of 0.000 ), which shows that if more people tend to enter or exit the building when the subject is within a distance of 4-10 $\mathrm{m}$ upstream of the entrance, the lateral deviation distance is larger. In addition, the correlation between $\Delta y$ and $P_{0,30}^{-150,-90}$ (with a $t$ value of 3.441 and a $p$ value of 0.001 ) is positive. This confirms that if more people move towards the wall in the $0^{\circ}$ to $30^{\circ}$ vision field on the sidewalk furthest from the wall, the deviation is larger. The correlation with $P_{0,30}^{-150,-90}$ can be caused by the collision avoidance strategy that passing pedestrians are more aware of collisions with those furthest from the wall towards the side nearest the wall and move to the side furthest from the wall to avoid potential collisions.

Lateral deviation $\left(R^{2}=0.384\right)$ :

$$
\Delta y=-0.236 y_{o}+0.497 F_{-10}^{-4}+5.018 P_{0,30}^{-150,-90}+1.535 \text {. }
$$

5.2.2. Longitudinal Deviation. The 20 variables found to be strongly correlated with $\Delta x$ are $x_{o}, \Delta y, F_{-20}^{-6}, F_{-20}^{-4}, F_{-18}^{-6}, F_{-18}^{-4}$, $F_{-16}^{-6}, F_{-16}^{-4}, F_{-14}^{-8}, F_{-14}^{-6}, F_{-14}^{-4}, F_{-12}^{-6}, F_{-12}^{-4}, F_{-10}^{-4}, E_{-4}^{6}, E_{-4}^{8}, E_{-2}^{8}, E_{0}^{8}$, $E_{2}^{8}$, and $P_{-90,-60}^{-30,30}$. After stepwise regression, $x_{o}, \Delta y, E_{-2}^{8}$, and $P_{-90,-60}^{-30,30}$ were incorporated into the regression model for predicting longitudinal deviation, as shown in equation (2).

For pedestrians passing the entrance area, if the deviation starts at a location further upstream, the longitudinal deviation is larger. This possibly indicates that pedestrians estimate in advance the space and time at which they need to 
TABLe 1: The $p$ value of the deviation process with lateral and longitudinal deviations.

\begin{tabular}{lcc}
\hline Variables & $\Delta y$ & $\Delta x$ \\
\hline$y_{o}$ & 0.000 & 0.037 \\
$x_{o}$ & 0.039 & 0.001 \\
$\Delta y$ & - & 0.000 \\
\hline
\end{tabular}

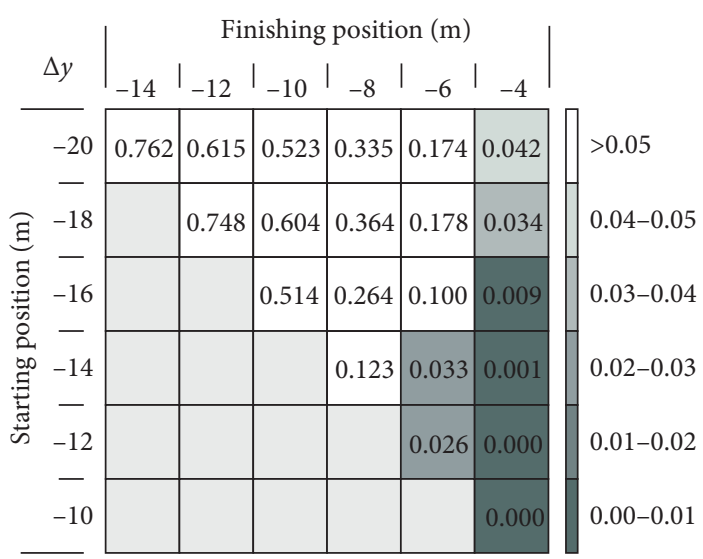

(a)

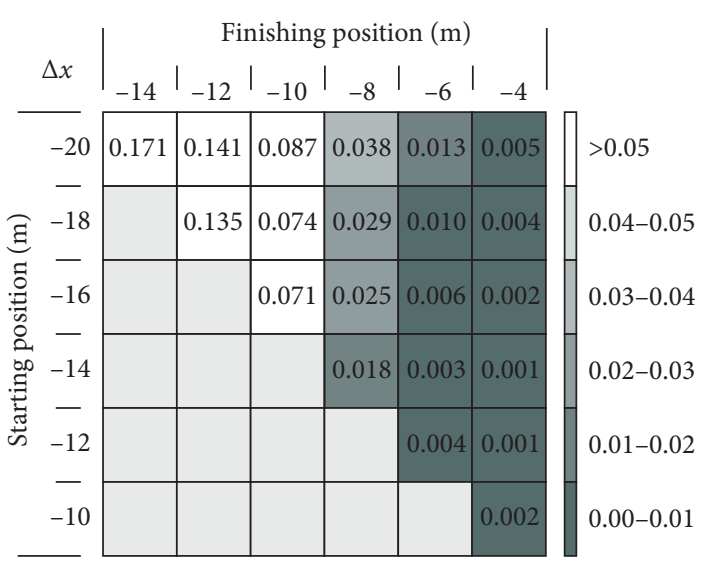

(b)

Figure 7: The $p$ value of entrance flow with lateral and longitudinal deviations.

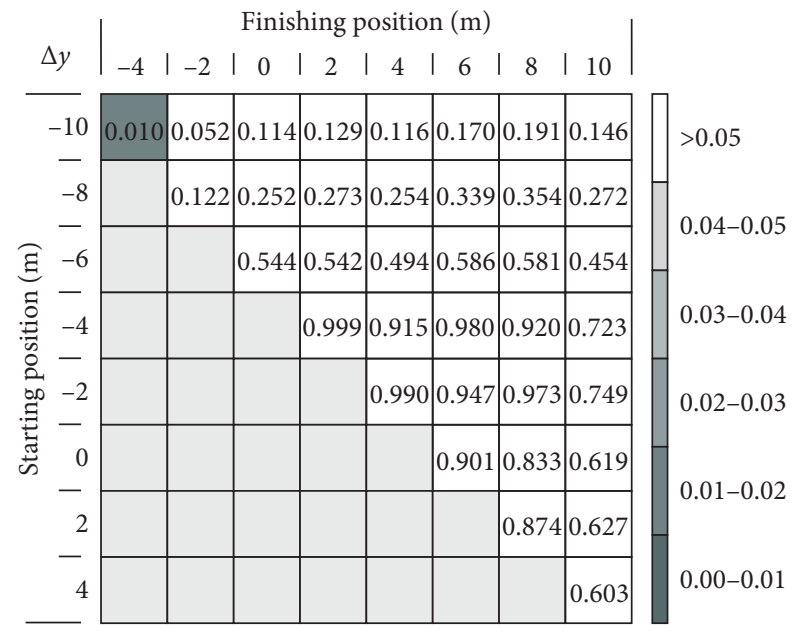

(a)

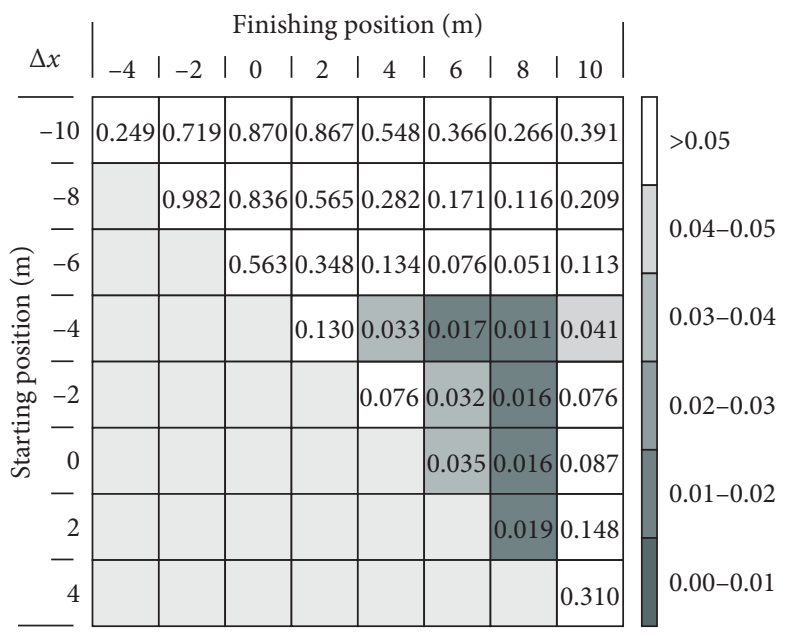

(b)

FIgURE 8: The $p$ value of entrance density with lateral and longitudinal deviations.

execute the deviation movement near the building entrance. In addition, when the lateral distance of deviation increases, the longitudinal distance required to complete the deviation also increases. Also, the entrance density between $2 \mathrm{~m}$ upstream of the entrance and $8 \mathrm{~m}$ downstream of the entrance when the passing pedestrian is being deviated has a negative correlation with the longitudinal deviation. This confirms that if more pedestrians are in the area a little downstream of the entrance, passing pedestrians will return to their desired path in advance without walking much further. Finally, $\Delta x$ has a negative correlation with $P_{-90,-60}^{-30,30}$ (with a $t$ value of
-4.186 and $p$ value of 0.000 ). That is, within the $-90^{\circ}$ to $-60^{\circ}$ field of vision on the side near the wall, if more pedestrians are walking along the wall, the longitudinal deviation distance is smaller. This implies that if more pedestrians are walking in the desired direction of passing pedestrians, they will follow instead of being further deviated.

Longitudinal deviation $\left(R^{2}=0.495\right)$ :

$$
\begin{aligned}
\Delta x= & -0.286 x_{o}+1.889 \Delta y-20.531 E_{-2}^{8} \\
& -1.997 P_{-90,-60}^{-30,30}+12.320 .
\end{aligned}
$$




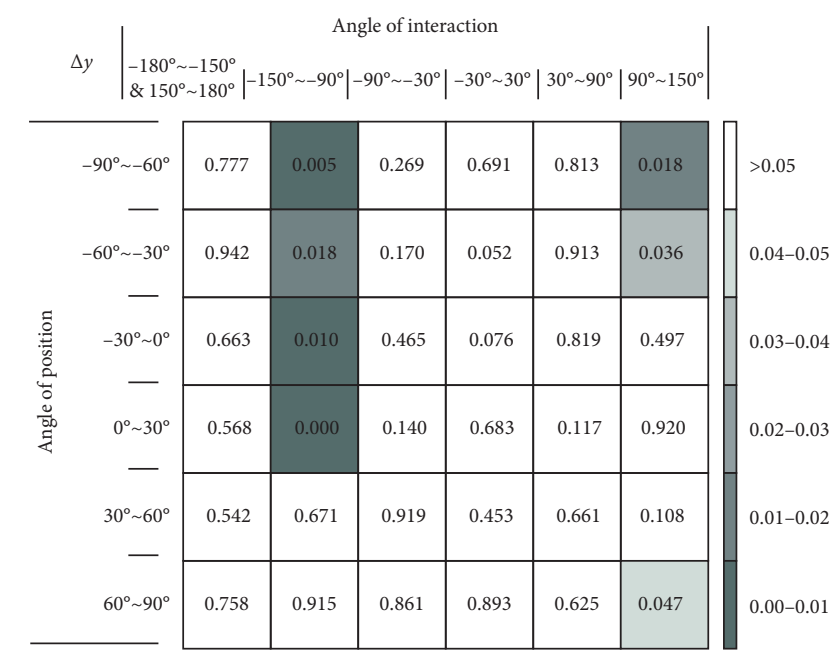

(a)

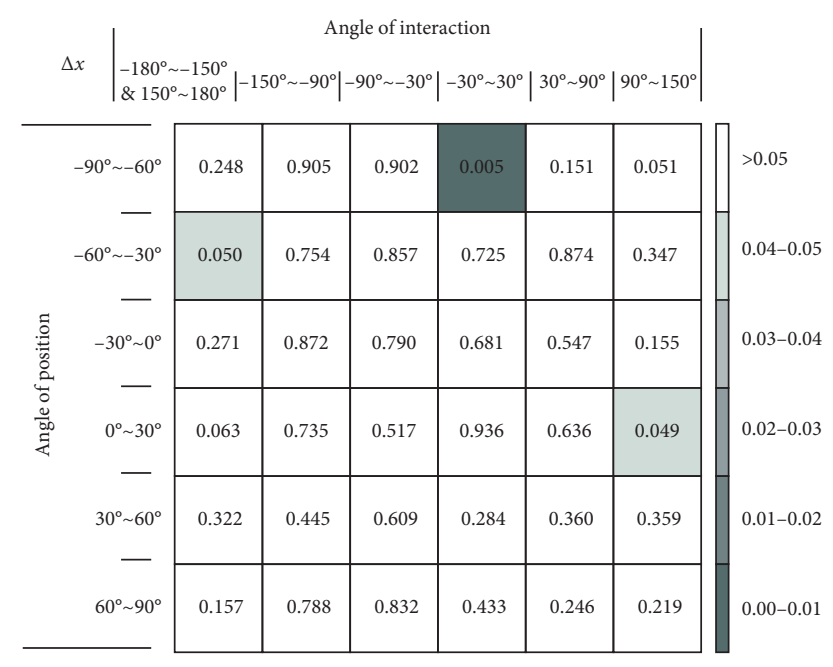

(b)

FIGURE 9: The $p$ value of the number of interacting pedestrians with lateral and longitudinal deviations.

\section{Conclusions}

In this study of pedestrian movement dynamics in a commercial pedestrian area, we analysed the impacts near a building's entrance and the flow rate at the entrance on the deviation behaviour of pedestrians. Footage recorded using a UAV camera was analysed to derive a dataset featuring pedestrian trajectories near a building entrance. The deviation behaviour was quantified using a two-step analysis process consisting of one-to-one correlation tests and a stepwise linear regression modelling procedure.

We showed that pedestrians passing a building entrance deviate from their intended path. In particular, the starting position of the deviation, flow rate at the entrance, density near the entrance, and walking pedestrians near the subject significantly affect the lateral and longitudinal deviation of the pedestrians that pass through the building's entrance area.

In particular, pedestrians walk near the wall show more deviation, on average, than pedestrians walking farther from the wall. Moreover, if the lateral deviation increases, the longitudinal deviation distance also increases. The starting position of the deviation affects the longitudinal deviation. Furthermore, the lateral deviation distances positively correlated with the flow rate at the entrance. Finally, the movement dynamics surrounding the subjects strongly affected the deviation behaviour. Depending on the locations and movement directions of the pedestrians interacting with the subjects, the deviation distance increases or decreases, which coincides with certain self-organisation behaviour. Passing pedestrians are influenced by collisions with pedestrians moving from paths far from the wall towards the side near the wall and show more lateral deviation, which can form a temporarily circulating flow. Because the side near the entrance is more cluttered and has a reduced visual scope, the pedestrians will choose the side farthest from the entrance. The decrease of lateral deviation influenced by pedestrians walking along the wall on the near side of the wall can be a result of lane formation. When the most chaotic area has been traversed, pedestrians moving in a similar direction as the others will be attracted, to form a lane. If the lane is nearer to the wall, they will complete the deviation process earlier.

\section{Implications of This Study for Designing Pedestrian Urban Spaces}

In this study, we established that the movement of pedestrians near building facades is affected by the presence of an entrance. In particular, the inflow and outflow of the building are found to "push" passing pedestrians away from the entrance. As such, near busy shopping mall entrances, the pedestrian density may be less evenly distributed than originally expected. This implies that, next to an "empty" zone directly in front of an entrance, a more densely populated zone further away from the entrance is created, which may cause a potential decrease in the level-of-service of commercial pedestrian spaces. More importantly, however, this effect may decrease the capacity of commercial spaces, posing a potential safety risk. To address these two issues, more space should be reserved for pedestrian movements near the entrances of attractive buildings (i.e., buildings with steady, high inflow, and outflow rates). In addition, a study conducted by Hou and Zheng [49] serves as an indication that building design can be used to improve the distribution of pedestrians in the vicinity of buildings and help balance pedestrian flows near a building.

Our findings have strong implications for the long term, where building owners, building functions, or building designs are expected to change. During these processes, the locations of entrances and the attractions of the buildings might change. These also cause changes in the walking dynamics in the vicinity of such a site. Thus, the design of pedestrian spaces in commercial areas is not a static onetime action but an iterative process in which the suitability of 
pedestrian spaces should be assessed whenever such changes occur at a site.

Although this research mainly focused on the walking dynamics near the entrance of a commercial building, the general concept behind this change in movement dynamics is applicable to a wider field. In essence, the deviating movement of the pedestrian of focus is a risk-avoidance strategy triggered by a chaotic walking environment during the period when the pedestrian can see the entrance. Similar strategies are probably adopted in other scenarios to cope with the chaotic walking environment and lack of visibility in certain parts of pedestrian spaces. We believe that it is essential to study these anticipatory risk-avoidance strategies further, develop generic models that include these strategies, and implement these behavioural models in contemporary pedestrian simulations to reproduce pedestrian spaces more accurately in areas where the visibility and complexity of pedestrian flows are challenging.

\section{Data Availability}

The data used to support the findings of this study are available from the corresponding author upon request.

\section{Disclosure}

A previous version of this research was presented at the 2020 Transportation Research Board Annual Meeting.

\section{Conflicts of Interest}

The authors declare that they have no conflicts of interest.

\section{Acknowledgments}

The authors would like to thank Ying Liu, Yongjie Pan, and Mengmeng Li for their assistance in conducting this research. This work was supported by the China Scholarship Council (Grant no. 201806120266), the research program "Allegro: Unravelling slow mode travelling and traffic: with innovative data toward a new transportation and traffic theory for pedestrians and bicycles" (Grant agreement no. 669792), and National Natural Science Foundation of China (Grant no. 51878202).

\section{References}

[1] Y. Yoshimura, A. Amini, S. Sobolevsky, J. Blat, and C. Ratti, "Analysis of pedestrian behaviors through non-invasive bluetooth monitoring," Applied Geography, vol. 81, pp. 43-51, 2017.

[2] V. X. Gong, W. Daamen, A. Bozzon, and S. P. Hoogendoorn, "Estimate sentiment of crowds from social media during city events," Transportation Research Record: Journal of the Transportation Research Board, vol. 26, no. 11, pp. 836-850, 2019.

[3] X. Xu, X. Zhou, and J. Chen, "Risk analysis of stampede accidents in large business district," International Symposium on Engineering Management, vol. 2009, pp. 115-119, 2009.

[4] M. Hu, Z. Fang, S. Liu, W. Chen, and B. Yang, "Study on evolution mechanism of fateful stampede accident based on graphical evaluation and review technique," in Proceedings of the 2010 IEEE International Conference on Systems, Man and Cybernetics, pp. 2976-2978, Istanbul, Turkey, October 2010.

[5] S. P. Hoogendoorn, F. L. M. Van Wageningen-Kessels, W. Daamen, and D. C. Duives, "Continuum modelling of pedestrian flows: from microscopic principles to self-organised macroscopic phenomena," Physica A: Statistical Mechanics and Its Applications, vol. 416, no. 15, pp. 684-694, 2014.

[6] D. C. Duives, W. Daamen, and S. P. Hoogendoorn, "Continuum modelling of pedestrian flows-part 2: sensitivity analysis featuring crowd movement phenomena," Physica A: Statistical Mechanics and Its Applications, vol. 447, no. 1, pp. 36-48, 2016.

[7] Y. Xiaoxia, W. Daamen, S. P. Hoogendoorn, H. Dong, and $\mathrm{X}$. Yao, "Dynamic feature analysis in bidirectional pedestrian flows," Chinese Physics B, vol. 25, no. 2, Article ID 028901, 2016.

[8] D. Helbing and P. Molnár, "Social force model for pedestrian dynamics," Physical Review E, vol. 51, no. 5, pp. 4282-4286, 1995.

[9] J. Zacharias, "Pedestrian behavior pedestrian behavior and perception in urban walking environments," Journal of Planning Literature, vol. 16, no. 1, pp. 3-18, 2001.

[10] K. Kirsten and D. Walton, "Field observations to determine the influence of population size, location and individual factors on pedestrian walking speeds," Ergonomics, vol. 51, no. 6, pp. 827-842, 2008.

[11] D. C. Duives, W. Daamen, and S. P. Hoogendoorn, "Proposition and testing of a conceptual model describing the movement of individual pedestrians within a crowd," Transportation Research Procedia, vol. 9, pp. 36-55, 2015.

[12] Y. Tanaboriboon, S. Hwa, and H. Chin, "Pedestrian characteristics study in Singapore," Journal of Transportation Engineering, vol. 112, no. 3, pp. 229-235, 1986.

[13] Y. Tanaboriboon and J. A. Guyano, "Analysis of pedestrian movements in Bangkok," Transportation Research Record, vol. 1294, pp. 52-56, 1991.

[14] W. Tian, W. Song, W. Lü, and Z. Fang, "Experiment and analysis on microscopic characteristics of pedestrian movement in building bottleneck," Science China Technological Sciences, vol. 54, no. 7, pp. 1730-1736, 2011.

[15] W. Boles, "The effect of density, sex, and group size upon pedestrian walking velocity," Man-Environment Systems, vol. 11, pp. 37-40, 1981.

[16] M. Moussaïd, N. Perozo, S. Garnier, D. Helbing, and G. Theraulaz, "The walking behaviour of pedestrian social groups and its impact on crowd dynamics," PLoS One, vol. 5, no. 4, Article ID e10047, 2010.

[17] C. H. Jazwinski and C. H. Walcheski, "At the mall with children: group size and pedestrian economy of movement," Environment and Behavior, vol. 43, pp. 363-386, 2011.

[18] S. Cao, J. Zhang, D. Salden, J. Ma, C. Shi, and R. Zhang, "Pedestrian dynamics in single-file movement of crowd with different age compositions," Physical Review E, vol. 94, no. 1, Article ID 012312, 2016.

[19] B. D. Hankin and R. A. Wright, "Passenger flow in subways," Journal of The Operational Research Society, vol. 9, no. 2, pp. 81-88, 1958.

[20] U. Weidmann, “Transporttechnik der Fußg”anger: transporttechnische eigenschaften des Fußgängerverkehrs, literaturauswertung," IVT Schriftenreihe, vol. 90, pp. 1-109, 1993.

[21] W. Daamen and S. P. Hoogendoorn, "Experimental research of pedestrian walking behavior," Transportation Research 
Record: Journal of the Transportation Research Board, vol. 1828, no. 1, pp. 20-30, 2003.

[22] A. Seyfried, B. Steffen, W. Klingsch, and M. Boltes, "The fundamental diagram of pedestrian movement revisited," Journal of Statistical Mechanics: Theory and Experiment, vol. 2005, no. 10, pp. 1-13, 2005.

[23] J. Zacharias, "Path choice and visual stimuli: signs of human activity and architecture," Journal of Environmental Psychology, vol. 21, no. 4, pp. 341-352, 2001.

[24] Y. Qu, Y. Xiao, J. Wua, T. Tang, and Z. Gao, "Modeling detour behavior of pedestrian dynamics under different conditions," Physica A: Statistical Mechanics and Its Applications, vol. 492, pp. 1153-1167, 2018.

[25] T. Kretz, A. Grünebohm, M. Kaufman, F. Mazur, and M. Schreckenberg, "Experimental study of pedestrian counterflow in a corridor," Journal of Statistical Mechanics: Theory and Experiment, vol. 2006, no. 10, Article ID P10001, 2006.

[26] S. Wong, M. Asce, W. Leung et al., "Bidirectional pedestrian stream model with oblique intersecting angle," Journal of Transportation Engineering, vol. 136, no. 3, pp. 234-242, 2010.

[27] S. Cao, A. Seyfried, J. Zhang, S. Holl, and W. Song, "Fundamental diagrams for multidirectional pedestrian flows," Journal of Statistical Mechanics: Theory and Experiment, vol. 201, no. 3, Article ID 033404, 2017.

[28] M. Zhou, H. Dong, F. Wang, Q. Wang, and X. Yang, "Modeling and simulation of pedestrian dynamical behavior based on a fuzzy logic approach," Information Sciences, vol. 360, pp. 112-130, 2016.

[29] S. Haq, "Where we walk is what we see: foundational concepts and analytical techniques of space syntax," Herd-Health Environments Research \& Design Journal, vol. 12, no. 1, pp. 11-25, 2019.

[30] D. Helbing, L. Buzna, A. Johansson, and T. Werner, "Selforganized pedestrian crowd dynamics: experiments, simulations, and design solutions," Transportation Science, vol. 39, no. 1, pp. 1-24, 2005.

[31] M. Moussaïd, D. Helbing, S. Garnier, A. Johansson, M. Combe, and G. Theraulaz, "Experimental study of the behavioural mechanisms underlying self-organization in human crowds," Proceedings of The Royal Society B, vol. 276, no. 1668, pp. 2755-2762, 2009.

[32] J. Ma, W. Song, Z. Fang, S. Lo, and G. Liao, "Experimental study on microscopic moving characteristics of pedestrians in built corridor based on digital image processing," Building and Environment, vol. 45, no. 10, pp. 2160-2169, 2010.

[33] H. Oppewal and H. Timmermans, "Modeling consumer perception of public space in shopping centers," Environment and Behavior, vol. 31, no. 1, pp. 45-65, 1999.

[34] R. Meziani and H. Ali Hussien, "A study on the space layout and configuration of shopping malls in relation to pedestrian movement behavior-case of UAE," International Review for Spatial Planning and Sustainable Development, vol. 5, no. 3, pp. 53-65, 2017.

[35] S. E. M. Jansen, A. Toet, and P. J. Werkhoven, "Human locomotion through a multiple obstacle environment: strategy changes as a result of visual field limitation," Experimental Brain Research, vol. 212, no. 3, pp. 449-456, 2011.

[36] B. R. Fajen and W. H. Warren, "Behavioral dynamics of steering, obstable avoidance, and route selection," Journal of Experimental Psychology: Human Perception and Performance, vol. 29, no. 2, pp. 343-362, 2003.

[37] C. Chen, "Attention theory-based agent system: using shopping street design simulation as an example," Journal of the Chinese Institute of Engineers, vol. 34, no. 1, pp. 155-168, 2011.

[38] J. Matthis and B. Fajen, "Visual control of foot placement when walking over complex terrain," Journal of Experimental Psychology: Human Perception and Performance, vol. 40, no. 1, pp. 106-115, 2013.

[39] O. Severiukhina, D. Voloshin, M. Lees, and V. Karbovskii, "The study of the influence of obstacles on crowd dynamics," Procedia Computer Science, vol. 108, pp. 215-224, 2017.

[40] T. Ezaki, D. Yanagisawa, K. Ohtsukac, and K. Nishinari, "Simulation of space acquisition process of pedestrians using proxemic floor field model," Physica A: Statistical Mechanics and Its Applications, vol. 391, pp. 291-299, 2012.

[41] S. Ham and G. Lee, "Space-connector model for representing the geometric properties of connections between spaces," Architectural Science Review, vol. 61, no. 6, pp. 429-442, 2018.

[42] T. Kitade, S. Satake, T. Kanda, and M. Imai, "Understanding suitable locations for waiting," in Proceedings of the ACM IEEE International Conference on Human-Robot Interaction, pp. 57-64, Portland, OR, USA, March 2013.

[43] J. Zacharias, T. Bernhardt, and L. D. Montigny, "Computersimulated pedestrian behavior in shopping environment," Journal of Urban Planning and Development, vol. 131, no. 3, pp. 195-200, 2005.

[44] M. Huber, Y.-H. Su, M. Krüger, K. Faschian, S. Glasauer, and J. Hermsdörfer, "Adjustments of speed and path when avoiding collisions with another pedestrian," PLoS One, vol. 9, no. 2, pp. 1-13, 2014.

[45] A. M. C. B. Silva, J. da Cunha, and J. Pedro, "Estimation of pedestrian walking speeds on footways," Proceedings of the Institution of Civil Engineers-Municipal Engineer, vol. 167, no. 1, pp. 32-43, 2014.

[46] A. Johansson, D. Helbing, and P. K. Shukla, "Specification of the social force pedestrian model by evolutionary adjustment to video tracking data," Advances in Complex Systems, vol. 10, pp. 271-288, 2007.

[47] Y. Feng, D. Duives, W. Daamen, and S. Hoogendoorn, "Data collection methods for studying pedestrian behaviour: a systematic review," Building and Environment, vol. 187, Article ID 107329, 2021.

[48] X. Ji, J. Zhang, Y. Hu, and B. Ran, "Pedestrian movement analysis in transfer station corridor: velocity-based and acceleration-based," Physica A: Statistical Mechanics and Its Applications, vol. 450, pp. 416-434, 2016.

[49] X. Hou and L. Zheng, "Simulation of pedestrian flow in traditional commercial streets based on space syntax," Procedia Engineering, vol. 205, pp. 1344-1349, 2017. 